\title{
Altered expression of myosin heavy chain in the vastus lateralis muscle in patients with COPD
}

\author{
F. Maltais*, M.J. Sullivan ${ }^{+}$, P. LeBlanc*, B.D. Duscha ${ }^{+}$, F.H. Schachat ${ }^{+}$, \\ C. Simard", J.M. Blank ${ }^{+}$, J. Jobin*
}

Altered expression of myosin heavy chain in the vastus lateralis muscle in patients with COPD. F. Maltais, M.J. Sullivan, P. LeBlanc, B.D. Duscha, F.H. Schachat, C. Simard, J.M. Blank, J. Jobin. (C) ERS Journals Ltd 1999.

ABSTRACT: This study was designed to further characterize peripheral skeletal muscle alterations in patients with chronic obstructive pulmonary disease (COPD) and to evaluate the possible relationship between myosin heavy chain (MyoHC) isoform expression and exercise tolerance in these individuals.

MyoHC composition from biopsy of the vastus lateralis muscle was examined in 12 COPD patients (forced expiratory volume in one second $($ FEV1) $=31 \pm 9 \%$ predicted, peak oxygen consumption $\left.\left(V^{\prime} \mathrm{O}_{2}\right)=15 \pm 4 \mathrm{~mL} \cdot \mathrm{kg}^{-1} \cdot \mathrm{min}^{-1}\right)$ and 10 age-matched normal male subjects (peak $V^{\prime} \mathrm{O}_{2}=\mathbf{2 0} \pm 5 \mathrm{~mL} \cdot \mathrm{kg}^{-1} \cdot \mathrm{min}^{-1}$ ).

The proportion of MyoHC type I was smaller in COPD than in normals $(27 \pm 17 \%$ versus $41 \pm 9 \%, \mathrm{p}<0.05)$ with an increase in MyoHC type IIa $(51 \pm 15 \%$ versus $39 \pm 9 \%$, $p<0.05)$ and the proportion of MyoHC type IIx being comparable between both groups. A significant relationship was found between peak $V^{\prime} \mathrm{O}_{2} \mathrm{~mL} \cdot \mathrm{kg}^{-1} \cdot \mathrm{min}^{-1}$ and FEV1 \% pred $(r=0.91, p<0.0001)$ and the percentage of MyoHC type $\mathrm{I}(\mathrm{r}=0.61$, $\mathrm{p}=0.016$ ). In stepwise multiple regression, only FEV1 \% pred was found to be a significant determinant of peak $V^{\prime} O_{2}(p<0.0001)$. This variable explained $83 \%$ of the total variance of peak $V^{\prime} \mathrm{O}_{2}$.

In summary, this study showed considerable modifications in the phenotypic expression of the myosin heavy chain in the vastus lateralis muscle in patients with chronic obstructive pulmonary disease. An independent effect of myosin heavy chain expression on exercise capacity was not found. These results suggest that chronic inactivity and muscle deconditioning may not be the sole factors explaining peripheral muscle dysfunction in patients with chronic obstructive pulmonary disease.

Eur Respir J 1999; 13: 850-854.
*Unité de recherche, Institut de Cardiologie et de Pneumologic de Québec, Québec, Canada. ${ }^{+}$Dept of Medicine, Division of Cardiology, Duke University Medical Center, Durham, NC, USA and †Dept of éducation physique, Université Laval, Québec, Canada.

Correspondence: F. Maltai
Centre de Pneumologie
Hôpital Laval
2725 Chemin Ste-Foy
Ste-Foy, QC
GIV 4G5
Canada
Fax: 4186564762

Fax: 4186564762

Keywords: Exercise lung disease

skeletal muscle

Received: June 211998

Accepted after revision November 171998

This study was supported in part by the Fonds de la recherche en santé du Québec, by la fondation JD Bégin, Université Laval, and by Bayer Corporation. F. Maltais is a clinician-scientist of the Fonds de la recherche en santé du Québec.
Exercise intolerance is a major consequence of chronic obstructive pulmonary disease (COPD). Although most studies have concentrated on limitation in ventilation and gas exchange, the large variation in exercise capacity for a given level of airflow obstruction and the persistence of a low exercise capacity after lung transplantation suggest that other factors are involved in explaining exercise intolerance in COPD [1, 2].

The possible role of altered peripheral muscle function in exercise intolerance in patients with COPD is currently being investigated. Compared to age-matched normal subjects, peripheral muscle weakness, reduction in oxidative enzyme activities and a low type I fibre proportion with a reciprocal increase in type IIb fibre proportion in the vastus lateralis muscle have been reported in patients with moderate-to-severe COPD [3-8]. A recent study has compared the myosin heavy chain (MyoHC) composition of the vastus lateralis muscle in 22 patients with moderate COPD and 10 young normal subjects [9]. A significant increase in type IIb MyoHC was found in their patients, while the proportion of the other types of MyoHC (I and IIa) was comparable between both groups. This finding of a normal proportion of type I MyoHC in these COPD patients contrasts with previous reports which have shown, using histochemical fibre typing, a marked reduction in the proportion of type I fibres in patients with COPD [3, 4, 8]. However, the patients studied by SATTA et al. [9] were less severely obstructed compared to those included in earlier studies. It is, therefore, conceivable that their peripheral muscle functions were better preserved.

An important issue concerning the peripheral muscle dysfunction in patients with COPD, is whether it is entirely due to chronic inactivity and muscle deconditioning or if there is an intrinsic muscle disorder. Because patients with COPD and normal subjects were not matched for exercise tolerance and physical activities, previous studies do not help to differentiate these two possibilities.

The present study was designed to characterize the $\mathrm{MyoHC}$ isoform expression of the vastus lateralis muscle in patients with severe COPD and to study its potential impact on exercise tolerance. In order to minimize the effect of fitness level in explaining the possible differences in MyoHC composition between COPD patients and their controls, normal subjects with sedentary habits were carefully selected to participate in the study. The objectives of this protocol were achieved by obtaining biopsy specimens of the vastus lateralis muscle in 12 patients with COPD and in 10 sedentary, age-matched normal subjects. 


\section{Subjects and methods}

\section{Subjects}

Twelve male patients with COPD were recruited at the Hôpital Laval, Ste-Foy, Quebec, Canada to participate in this study. In each case, the diagnosis of COPD was based on previous or current smoking history and pulmonary function tests showing moderate-to-severe irreversible airflow obstruction (forced expiratory volume in one second (FEV1) $<60 \%$ of predicted value and FEV1/forced vital capacity $(\mathrm{FVC})<70 \%$ ). No clinical evidence of exercise-limiting cardiovascular or neuromuscular diseases were found in these patients. At the time of evaluation, they were in a stable condition with no exacerbation of their disease and/or use of systemic corticosteroid in the preceding two months. Ten healthy, age-matched nonsmoking males were recruited at Duke University, Durham, NC, USA and constituted the control group. These subjects had no cardiopulmonary dysfunction as indicated by history and physical examination, and none exhibited symptoms of lung or heart disease. None of these subjects were involved in regular aerobic exercise training, recreational activities, or heavy occupational duties. The research protocol was approved by the institutional ethics committees and a signed informed consent was obtained in each case

Pulmonary function tests. All COPD patients underwent standard pulmonary function testing including spirometry, lung volumes and carbon monoxide diffusing capacity of the lung ( $D \mathrm{~L}, \mathrm{CO})$ according to previously described guidelines [10]. Pulmonary function results were related to normal values of KNUDSON et al. [11], GoLDmAN and BeCKLAKe [12], and Cotes and Hall [13], respectively. In COPD, arterial blood gas was also analysed at rest for arterial oxygen tension $\left(\mathrm{Pa}_{\mathrm{a}} \mathrm{O}_{2}\right)$, arterial carbon dioxide tension $\left(\mathrm{Pa}_{\mathrm{a}} \mathrm{CO}_{2}\right)$, and $\mathrm{pH}$ determination.

Muscle biopsy. Percutaneous biopsies of the right vastus lateralis muscle were performed at mid-thigh level (15 $\mathrm{cm}$ above the patella) as described by BERGSTRÖM [14] and prior studies by the authors' group [7, 15]. Biopsy sites were anaesthetized with a $2 \%$ lidocaine solution, and $0.5-1 \mathrm{~cm}$ skin incisions were made through the skin and fascia lata. They were immediately frozen in isopentane cooled to freezing point with liquid nitrogen and stored at $-70^{\circ} \mathrm{C}$ until processing. All biopsy specimen were analysed at Duke University, Durham, NC, USA.

Exercise testing in normal subjects. All normal subjects underwent graded upright bicycle exercise to a symptom-limited maximum on a isokinetic ergometer (Fitron; Lurnex, Inc., Ronkonkona, NY, USA) as previously described [15]. The workload began at $25 \mathrm{~W}$ and advanced in 3-min stages of $25 \mathrm{~W}$. Expired gases were analysed continuously using a Sensormedics 4400 unit (Anaheim, CA, USA). Heart rate, minute ventilation $\left(V^{\prime} \mathrm{E}\right)$, oxygen consumption $\left(V^{\prime} \mathrm{O}_{2}\right)$, and carbon dioxide production $\left(V^{\prime} \mathrm{CO}_{2}\right)$ were measured at rest and during exercise.

Exercise testing in COPD patients. COPD patients were seated on an electrically braked ergocycle and connected to an expired gas analysing system through a mouthpiece. The gas analysing circuit consisted of a pneumo- tachograph, $\mathrm{O}_{2}$, and $\mathrm{CO}_{2}$ analysers and a mixing chamber (Quinton Qplex, A-H Robins Company, Seattle, WA, USA). After $5 \mathrm{~min}$ of rest, a progressive stepwise exercise test was performed up to individual's maximum capacity. Each exercise step lasted $1 \mathrm{~min}$, and increments of $10 \mathrm{~W}$ were used. Heart rate, $V^{\prime} \mathrm{E}, V^{\prime} \mathrm{O}_{2}, V^{\prime} \mathrm{CO}_{2}$, dyspnoea and leg fatigue scores on a modified Borg scale [16] were measured at rest and during exercise.

\section{Skeletal muscle and data analysis}

Sample preparation for sodium dodecylsulphate-polyacrylamide gel electrophoresis. Muscle biopsy samples ( $\sim 30 \mathrm{mg}$ each) were minced with razor blades on a chilled glass plate and transferred to microcentrifuge tubes. The samples were washed, resuspended twice in a rigor solution containing protease inhibitors $(0.1 \mathrm{mM}$ $\mathrm{NaCl}, 15 \mathrm{mM}$ Tris $\mathrm{pH} 8.0,2 \mathrm{mM} \mathrm{MgCl}_{2}, 4 \mathrm{mM}$ ethyleneglycol-bis-( $\beta$-aminoethylether)- $N, N, N^{\prime}, N^{\prime}$-tetraacetic acid (EGTA), $7 \mathrm{mM} \beta$-mercaptoethanol, $0.1 \mathrm{mM}$ phenylmethylsulphonyl fluoride (PMSF), $10 \mu \mathrm{g} \cdot \mathrm{L}^{-1}$ leupeptin, antipain and pepstatin and centrifuged at $300 \times g$ for 2 $\min$ at $4^{\circ} \mathrm{C}$ ). Following the initial washes, the pellets were resuspended in rigor solution containing $2 \%$ Triton X-100 (a detergent) to solubilize membrane-bound components, and then washed twice more in rigor buffer. Aliquots of myofibrils were dissolved in a sodium dodecylsulphate gel-loading buffer and frozen at $-80^{\circ} \mathrm{C}$.

MyoHC isoform resolution. MyoHC isoforms were resolved on $7.4 \%$ polyacrylamide gels containing $35 \%$ glycerol [17]. Following electrophoresis, gels were fixed in $15 \%$ acetic acid and stained with Coomassie Brilliant Blue G-250. MyoHC isoforms I, IIa and IIx were identified by order of migration according to ENNION et al. [18] and SMERDu et al. [19]. (Human type Ilb muscles fibres as identified by histochemistry are now known to express the IIx MyoHC isoform rather than the IIb isoform.) Gels were scanned electronically and relative proportions of $\mathrm{MyoHC}$ isoform were measured using National Institutes of Health (NIH) Image 1.60 for Macintosh (NIH, Bethesda, MD, USA) and Jandel Peakfit for Windows (SPSS, Chicago, IL, USA). Results were expressed as percentages of total MyoHC in each sample.

\section{Statistical analysis}

Values are reported as mean \pm SD. Intergroup data were compared using unpaired Student's t-tests. Maximum voluntary ventilation (MVV) was estimated by multiplying FEV1 by 35 [20]. Regression analyses were performed using the least square method to evaluate possible correlations between peak $V^{\prime} \mathrm{O}_{2}$, and $\mathrm{FEV}_{1} \%$ pred and the relative proportion of the three $\mathrm{MyoHC}$ isoforms. Using the variables significantly related to maximal exercise capacity, a stepwise multiple regression analysis was performed using peak $V^{\prime} \mathrm{O}_{2}$ as the dependent variable. A p-value of $<0.05$ was considered statistically significant.

\section{Results}

The characteristics of the experimental and control groups are given in table 1 . Age and body mass index 
Table 1. - Subject characteristics, pulmonary function tests and peak exercise data

\begin{tabular}{|c|c|c|}
\hline & $\begin{array}{c}\text { Normal subjects } \\
\qquad(\mathrm{n}=10)\end{array}$ & $\begin{array}{l}\text { COPD } \\
(n=12)\end{array}$ \\
\hline Age yrs & $61 \pm 6$ & $65 \pm 5$ \\
\hline Height $\mathrm{m}$ & $1.74 \pm 0.13$ & $1.67 \pm 0.09$ \\
\hline Weight $\mathrm{kg}$ & $83 \pm 12$ & $70 \pm 10^{*}$ \\
\hline Body mass index $\mathrm{kg} \cdot \mathrm{m}^{-2}$ & $28 \pm 4$ & $25 \pm 3$ \\
\hline FEV1 L & - & $0.87 \pm 0.24$ \\
\hline FEV $1 \%$ pred & - & $31 \pm 9$ \\
\hline FVC L & - & $2.5 \pm 0.6$ \\
\hline FVC $\%$ pred & - & $59 \pm 16$ \\
\hline TLC \% pred & - & $117 \pm 19$ \\
\hline$D \mathrm{~L}, \mathrm{CO} \%$ pred & - & $72 \pm 22$ \\
\hline$V^{\prime} \mathrm{O}_{2} \mathrm{~mL} \cdot \mathrm{kg}^{-1} \cdot \mathrm{min}^{-1}$ & $20 \pm 5$ & $15 \pm 4 *$ \\
\hline$V^{\prime} \mathrm{E} \mathrm{L} \cdot \mathrm{min}^{-\mathrm{T}}$ & $89 \pm 27$ & $37.5 \pm 11.9^{*}$ \\
\hline Heart rate beats $\cdot \min ^{-1}$ & $152 \pm 19$ & $135 \pm 11 *$ \\
\hline Heart rate $\%$ max pred & $82 \pm 11$ & $75 \pm 6$ \\
\hline
\end{tabular}

Data are presented as mean \pm SD. COPD: chronic obstructive pulmonary disease; FEV1: forced expiratory volume in one second; FVC: forced vital capacity; TLC: total lung capacity; $D \mathrm{~L}, \mathrm{CO}$ : carbon monoxide diffusing capacity of the lung; $V^{\prime} \mathrm{O}_{2}$ : oxygen consumption; $V^{\prime} \mathrm{E}$ : minute ventilation. ${ }^{*}: \mathrm{p}<0.05$.

were comparable in both groups. The experimental group had severe airflow obstruction with an FEV1 of $31 \pm 9 \%$ predicted, normal resting $P \mathrm{a}, \mathrm{O}_{2}(10.8 \pm 1.1 \mathrm{kPa})$ and $P \mathrm{a}, \mathrm{CO}_{2}$ $(5.5 \pm 0.5 \mathrm{kPa})$. All subjects completed the incremental exercise test to their maximal subjective capacity (table 1). The difference in peak $V^{\prime} \mathrm{O}_{2}$, between both groups was of small magnitude $\left(15 \pm 4 \mathrm{~mL} \cdot \mathrm{kg}^{-1} \cdot \mathrm{min}^{-1}\right.$ versus $20 \pm 5$ $\mathrm{mL} \cdot \mathrm{kg}^{-1} \cdot \mathrm{min}^{-1}$, for the COPD group and control group, respectively), but reached statistical significance $(p<0.05)$. At peak exercise, COPD had exhausted their ventilatory reserve $\left(V^{\prime} \mathrm{E} / \mathrm{MVV}=101 \pm 24 \%\right)$ while dyspnoea and leg fatigue Borg scores amounted to $7.7 \pm 1.8$ and $7.2 \pm 2.2$, respectively.

Figure 1 demonstrates the different migration rates of MyoHC type I, IIa, and IIx between two normal subjects and two COPD patients. The control biopsies (lanes A and B) exhibit the three MyoHC isoforms expressed in human skeletal muscle, type IIx, type IIa and type I. The COPD patient biopsies exhibit greater variation; some are comparable to controls (lane C), while others (lane D) exhibit very little MyoHC type I and an increase in the ratio of MyoHC type IIa relative to type IIx.

The group mean values for the relative content of MyoHC type I, IIa, and IIx in normal subjects and in patients with COPD are shown in figure 2. In COPD, a marked reduction in the percentage of MyoHC type I was found $(27 \pm 17 \%$ versus $41 \pm 9 \%$ in normals, $\mathrm{p}<0.05)$ with an increase in MyoHC type IIa (51 $\pm 15 \%$ versus $39 \pm 9 \%$, $\mathrm{p}<0.05)$ while the proportion of MyoHC type IIx was comparable between both groups $(22 \pm 16 \%$ versus $20 \pm$ $9 \%)$. No change in the ratio of myosin to actin or other myofibrillar proteins was observed. Thus, the ratio of the $\mathrm{MyoHC}$ to one another also represents their ratio to total myofibrillar proteins.

A significant relationship was found between peak $V^{\prime} \mathrm{O}_{2}$ $\mathrm{mL} \cdot \mathrm{kg}^{-1} \cdot \mathrm{min}^{-1}$ and FEV1 \% pred $(\mathrm{r}=0.91, \mathrm{p}<0.0001)$ and the percentage of MyoHC type I $(r=0.61, p=0.016)$. The percentage of MyoHC type I was significantly correlated to $\mathrm{FEV} 1 \%$ pred $(\mathrm{r}=0.66, \mathrm{p}=0.005)$. The other two MyoHC
A B C D

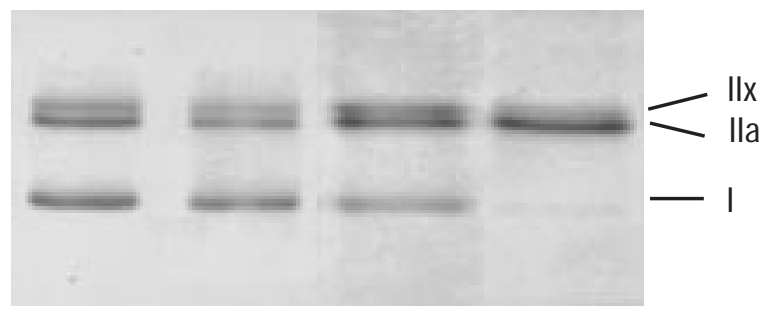

Fig. 1. - Electrophoretic separation of myosin heavy chain (MyoHC) isoforms from two normal subjects (lanes A and B) and two chronic obstructive pulmonary disease (COPD) patients (C and D). Homogenates prepared from age-matched sedentary controls and COPD patients biopsies were subjected to sodium dodecylsulphate-gycerol polyacrylamide gel electrophoresis to resolve the MyoHC isoforms. The control biopsies (lanes A and B) exhibit the three MyoHC isoforms expressed in human skeletal muscle, type IIx, type IIa and type I. The

isoforms were not significantly correlated with peak $V^{\prime} \mathrm{O}_{2}$ or with FEV1 \% pred. In stepwise multiple regression, only FEV1 \% pred was found to be a significant determinant of peak $V^{\prime} \mathrm{O}_{2}(\mathrm{p}<0.0001)$. This variable explained $83 \%$ of the total variance $\left(\mathrm{r}^{2}\right)$ of peak $V^{\prime} \mathrm{O}_{2}$.

\section{Discussion}

The present results demonstrate important alterations in relative MyoHC isoform expression in skeletal muscle in patients with severe COPD characterized by a marked reduction in MyoHC type I and a greater proportion of MyoHC type IIa when compared to normals subjects. These changes in the MyoHC isoforms expression differ to those previously reported in patients with moderate COPD [91]. Although the percentage of MyoHC type I was significantly related with peak $V^{\prime} \mathrm{O}_{2}$, in patients, it did not contribute independently to the total variance of peak $V^{\prime} \mathrm{O}_{2}$, in a stepwise regression analysis.

These findings are consistent with the results of a recent report from the authors' laboratory which showed, using adenosine triphosphatase (ATPase) histochemical fibre typing, a decrease in type I fibre in patients with severe

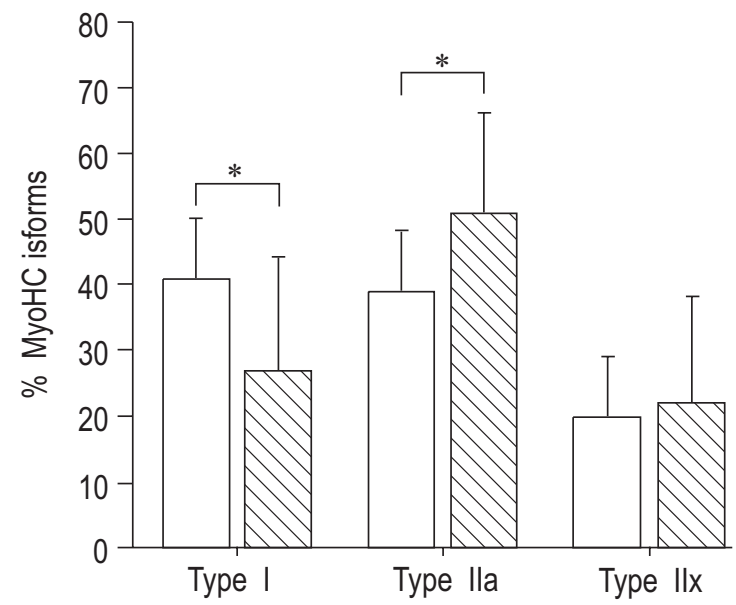

Fig. 2. - Mean \pm sD relative proportion of myosin heavy chain (MyoHC) isoforms in normal subjects $(\square)$ and in patients with chronic obstructive pulmonary disease (COPD; $\mathbb{Q}$ ). In COPD, a marked reduction in the percentage of MyoHC type I was found with an increase in relative content of MyoHC type IIa. *: p<0.05. 
COPD compared to age-matched normal subjects [8]. The fact that histochemical fibre typing and MyoHC expression determination in whole muscle provide similar information is in agreement with the notion that muscle fibres of a given type express mainly analogous $\mathrm{MyoHC}$ isoforms [21]. In the histochemical analysis, however, the relative proportion of histochemically type I fibre was slightly greater than that of MyoHC type I isoform reported here (34 versus $27 \%$ ) while the relative proportion of type IIb was smaller than that of MyoHC type IIx (15 versus 22\%). Important differences between histochemical fibre typing and the electrophoretic analysis of MyoHC expression in whole muscle biopsies may explain the slight variation in the results of these two studies. The greater proportion of MyoHC IIx with respect to histochemically type IIb fibres is most likely explained by the fact that some fibres labelled as type Ila using the histochemical staining, were actually coexpressing MyoHC type IIx and IIa. The coexpression of two or more $\mathrm{MyoHC}$ isoforms in individual fibres is difficult to recognize with classical ATPase histochemical fibre typing, which groups fibre into distinct categories. For this reason, direct quantification of the MyoHC isoforms profile in whole muscle specimen provides a more sensitive assessment of potential changes in muscle phenotypic expression of MyoHC than the classical histochemical method [22, 23]. Moreover, in contrast to the histochemical determination of fibre-type distribution, the total amount of a given MyoHC isofom is influenced by fibre size. Accordingly, the smaller relative proportion of MyoHC type I than of that of histochemically type I fibre in patients with COPD [21] may be explained by the smaller type I fibres than type II fibres in these individuals [8].

The MyoHC composition of the vastus lateralis muscle has recently been evaluated in patients with moderate COPD by SATTA et al. [9]. In contrast to the current findings, these authors reported a comparable proportion of MyoHC type I between normal subjects and COPD [9]. The different conclusions between the two studies can be explained by differences in the degree of airflow obstruction between the two sets of patients. This contention is strongly supported by the positive correlation between FEV $1 \%$ pred and the proportion of type I MyoHC reported in both studies.

The origins of the changes in the MyoHC phenotypic expression found in patients with COPD are still to be clarified. Data in animals and humans suggest that inactivity and muscle deconditioning are involved. In animals, muscle activation is one of the most potent stimuli which regulates the MyoHC gene expression [24]. Limb immobilization in mice reduces the expression of type I MyoHC in the skeletal muscle while stretch and force generation increase the expression of type I MyoHC and suppress that of type II MHC [24]. In human studies, after several years of permanent paralysis following spinal cord injury, the proportion of muscle fibres expressing type I MyoHC is extremely low [25]. However, under these conditions, the changes in the level of muscle activation were extreme and it is unclear to what extent these findings are relevant to the range of activity found in ambulatory humans. Nevertheless these studies support the role of inactivity in modifying the MyoHC phenotypic expression in patients with COPD. The similarity between the muscle changes associated with chronic inactivity in normal humans [26, 27] and those reported in patients with COPD, and the improvement in oxidative enzymes activity following aerobic training in COPD, also support the role of inactivity in explaining the peripheral muscle changes in these patients [28].

On the other hand, some observations support the notion that there are aetiologies other than inactivity to explain muscle changes in COPD. In the present study, important alterations in MyoHC isoform expression in the vastus lateralis muscle were found in patients with severe COPD compared to normal subjects despite modest differences in exercise capacity between the two groups. Although peak $V^{\prime} \mathrm{O}_{2}$ does not necessarily closely reflect the level of daily activities, it is unlikely that it was much different between normal subjects and patients with COPD, since no recreational activity or heavy occupational duties were reported by the normal subjects. Other evidence comes from a recent study performed in patients restricted to bed for over a year (following a cerebrovascular accident) [29]. In these patients with disuse atrophy, the changes in MyoHC phenotypic expression found in the nonparetic lower limb were opposite to those found in the current patients with COPD [29]. Other potential contributors to the development of peripheral muscle dysfunction in COPD include denutrition, chronic hypoxia [30], systemic use of corticosteroids, and low anabolic hormone levels [31]. Further studies are required to clarify the relative role of the different mechanisms potentially contributing to peripheral muscle dysfunction in COPD.

In this study, an independent effect of the skeletal muscle MyoHC expression on peak exercise capacity in patients with COPD was not found. This finding differs from others indicating that peripheral skeletal muscle function may directly influence exercise capacity in COPD $[5,6]$. In contrast to several studies which have found a weak-to-moderate relationship between FEV1 and maximal exercise capacity $\left(\mathrm{r}^{2} \sim 0.30-0.40\right)[6,32]$, the total variance in peak $V^{\prime} \mathrm{O}_{2}$ in the current patients was largely explained by the FEV $1 \%$ pred $\left(r^{2}=0.83\right)$. As a result, the probability to find another factor that significantly improves the prediction model of peak $V^{\prime} \mathrm{O}_{2}$, was substantially reduced. Other important determinants of the muscle aerobic capacity, such as muscle enzyme activities and capillarization which are altered in COPD [8] and may also influence exercise performance, were not evaluated in the present study. Therefore, the findings of this study that skeletal muscle MyoHC did not contribute independently to the total variance of peak $V^{\prime} \mathrm{O}_{2}$ in stepwise regression analysis should not be interpreted as indicating that peripheral muscle function does not play a role in exercise limitation in patients with COPD.

In summary, this study showed considerable modifications in the phenotypic expression of myosin heavy chains in the vastus lateralis muscle in patients with severe chronic obstructive pulmonatory disease compared to sedentary normal subjects. These modifications, which differ to those previously reported in patients with moderate lung disease, suggest that chronic inactivity and muscle deconditioning may not be the sole factors explaining peripheral muscle dysfunction in chronic obstructive pulmonary disease. Further research should be carried out to clarify the mechanisms underlying the development of peripheral muscle dysfunction in patients with chronic obstructive pulmonary disease. 
Acknowledgements. The authors thank $\mathrm{M}$ Bélanger, M-J. Breton, J-F. Doyon, and F. Whittom for their technical assistance and $\mathrm{S}$. Simard for his statistical assistance.

\section{References}

1. Jones NL, Killian KJ. Limitation of exercise in chronic airway obstruction. In: Cherniack NS, ed. Chronic Obstructive Pulmonary Disease. Philadelphia, W.B. Saunders; 1991; pp. 196-206.

2. Williams TJ, Patterson GA, Mcclean PA, Zamel N, Maurer JR. Maximal exercise testing in single and double lung transplant recipients. Am Rev Respir Dis 1992; 145: 101-105.

3. Jakobsson P, Jorfeldt L, Brundin A. Skeletal muscle metabolites and fibre types in patients with advanced chronic obstructive pulmonary disease (COPD), with and without chronic respiratory failure. Eur Respir J 1990; 3: 192-196.

4. Hildebrand IL, Sylvén C, Esbjornsson M, Hellstrom K, Jansson E. Does hypoxaemia induce transformations of fiber types? Acta Physiol Scand 1991; 141: 435-439.

5. Hamilton AL, Killian KJ, Summers E, Jones NL. Muscle strength, symptom intensity and exercise capacity in patients with cardiorespiratory disorders. Am J Respir Crit Care Med 1995; 152: 2021-2031.

6. Gosselink R, Troosters T, Decramer M. Peripheral muscle weakness contributes to exercise limitation in COPD. Am J Respir Crit Care Med 1996; 153: 976-980.

7. Maltais F, Simard AA, Simard C, Jobin J, Desgagnés P, Leblanc P. Oxidative capacity of the skeletal muscle and lactic acid kinetics during exercise in normal subjects and in patients with COPD. Am J Respir Crit Care Med 1996; 153: 288-293.

8. Whittom F, Jobin J, Simard PM, et al. Histochemical and morphological characteristics of the vastus lateralis muscle in COPD patients. Comparison with normal subjects and effects of exercise training. Med Sci Sports Exerc 1998; 30: 1467-1474.

9. Satta A, Migliori GB, Spanevello A, et al. Fibre types in skeletal muscles of chronic obstructive pulmonary disease patients related to respiratory function and exercise tolerance. Eur Respir J 1997; 10: 2853-2860.

10. American Thoracic Society. Standards for the diagnosis and care of patients with chronic obstructive pulmonary disease (COPD) and asthma. Am Rev Respir Dis 1987; 136: 225-244.

11. Knudson RJ, Slatin RC, Lebowitz MD, Burrows B. The maximal expiratory flow-volume curve. Normal standards, variability and effects of age. Am Rev Respir Dis 1976; 113: 587-600.

12. Goldman HI, Becklake MR. Respiratory function tests: normal values at median altitudes and the prediction of normal results. Am Rev Tuberc 1959; 79: 454-467.

13. Cotes JE, Hall AM. The transfer factors for the lung; normal values in adults. In: Arcangeli P, ed. Normal Values for Respiratory Function in Man. Torino, Panminerva Medica, 1970; pp. 327-343.

14. Bergström J. Muscle electrolytes in man. Determination by neutron activation analysis on needle biopsy specimens. A study on normal subjects, kidney patients and patients with chronic diarrhoea. Scand J Clin Lab Invest 1962; 14: 1-110.

15. Sullivan MJ, Green HJ, Cobb FR. Skeletal muscle biochemistry and histology in ambulatory patients with long-term heart failure. Circulation 1990; 81: 518-527.

16. Borg G. Psychophysical bases of perceived exertion. Med Sci Sports Exer 1982; 14: 377-381.

17. Talmadge RJ, Roy RR. Electrophoretic separation of rat skeletal muscle myosin heavy-chain isoforms. $J$ Appl Physiol 1993; 75: 2337-2340.

18. Ennion S, Pereira JS, Sargeant AJ, Young A, Goldspink G. Characterization of human skeletal muscle fibres according to the myosin heavy chain they express. $J$ Muscle Res Cell Motility 1995; 16: 35-43.

19. Smerdu V, Karsch-Mizrachi I, Campione M, Leinwand L, Schiaffino S. Type $2 \mathrm{X}$ myosin heavy chain transcripts are expressed in type 2B fibres of human skeletal muscle. $\mathrm{Am}$ J Physiol 1994; 267: C1723-C1728.

20. Clark TJH, Freedman S, Campbell EJM, Winn R. The ventilatory capacity of patients with chronic airway obstruction. Clin Sci 1969; 36: 307-316.

21. Adams GR, Hather BM, Baldwin KM, Dudley GA. Skeletal muscle myosin heavy chain composition and resistance training. J Appl Physiol 1993; 74: 911-915.

22. Staron RS, Johnson P. Myosin polymorphism and differential expression in adult human skeletal muscle. Comp Biochem Physiol 1993; 106: 463-475.

23. Sullivan VK, Powers SK, Criswell DS, Turmer N, Larochelle JS, Lowenthal D. Myosin heavy chain composition in young and old rat skeletal muscle: effects of endurance training. J Appl Physiol 1995; 78: 2115-2120.

24. Goldspink G, Scutt A, Loughna PT, Wells DJ, Jaenicke T, Gerlach GF. Gene expression in skeletal muscle in response to stretch and force generation. Am J Physiol 1992; 262: R356-R363.

25. Andersen JL, Mohr T, Biering-Sorensen F, Galbo H, Kjaer M. Myosin heavy chain isoform transformation in single fibres from $\mathrm{m}$. vastus lateralis in spinal cord injured individuals: effects of long-term functional electrical stimulation (FES). Pflügers Arch 1996; 431: 513-518.

26. Booth FW, Gollnick PD. Effects of disuse on the structure and function of skeletal muscle. Med Sci Sports Exer 1983; 15: 415-420.

27. Larsson L, Ansved T. Effects of long-term physical training and detraining on enzyme histochemical and functional skeletal muscle characteristics in man. Muscle Nerve 1985; 8: 714-722.

28. Maltais F, Leblanc P, Simard C, et al. Skeletal muscle adaptation to endurance training in patients with chronic obstructive pulmonary disease. Am J Respir Crit Care Med 1996; 154: 442-447.

29. Vescovo G, Serafini F, Facchin L, et al. Specific changes in skeletal muscle myosin heavy chain composition in cardiac failure: differences compared with disuse atrophy as assessed on microbiopsies by high resolution electrophoresis. Heart 1996; 76: 337-343.

30. Green HJ, Sutton JR, Cymerman A, Young PM, Houston CS. Operation Everest II: adaptations in human skeletal muscle. J Appl Physiol 1989; 66: 2454-2461.

31. Kamischke A, Kemper DE, Castel MA, et al. Testosterone levels in men with chronic obstructive pulmonary disease with or without glucocorticoid therapy. Eur Respir J 1998; 11: 41-45.

32. Wijkstra PJ, Ten Vergert EM, Van der Mark TW, et al. Relation of lung function, maximal inspiratory pressure, dyspnoea, and quality of life with exercise capacity in patients with chronic obstructive pulmonary disease. Thorax 1994; 49: 468-472. 